\begin{tabular}{|c|c|}
\hline Title & $\begin{array}{l}\text { X-ray Photoelectron Spectroscopy Study of Silicon Interlayer Based Surface Passivation for A IGaA s/GaA s Quantum } \\
\text { Structures on (111) B Surfaces }\end{array}$ \\
\hline Author(s) & A kazawa, Masamichi; Shiozaki, Nanako; Hasegawa, Hideki \\
\hline Citation & $\begin{array}{l}\text { Journal de Physique IV, 132, 95-99 } \\
\text { https://doi.org/10.1051/jp4:2006132019 }\end{array}$ \\
\hline Issue Date & 2006 \\
\hline Doc URL & http:/hdl .handle.net/2115/8495 \\
\hline Rights & (c) EDP Sciences 2006 \\
\hline Type & article (author version) \\
\hline File Information & Tu-B-1730-0_A kazawa_revised..pdf \\
\hline
\end{tabular}

Instructions for use 


\title{
X-ray Photoelectron Spectroscopy Study of Silicon Interlayer Based Surface Passivation for AIGaAs/GaAs Quantum Structures on (111) B Surfaces
}

\author{
Masamichi Akazawa, Nanako Shiozaki and Hideki Hasegawa \\ Research Center for Integrated Quantum Electronics and Graduate School of Information \\ Science and Technology, Hokkaido University, N-13, W-8, Sapporo 060-8628, Japan.
}

\begin{abstract}
Applicability of the Si interface control layer (Si ICL)-based surface passivation to GaAs and AlGaAs (111)B surfaces was investigated. An in-situ X-ray photoelectron spectroscopy (XPS) study confirmed formation of the intended passivation structure. MBE grown GaAs and AlGaAs (111)B surfaces showed strong Fermi level pinning. After Si ICL growth, large shifts of the surface Fermi level position were observed. Photoluminescence (PL) measurements were also used to examine the surfaces of $\mathrm{AlGaAs} / \mathrm{GaAs}$ quantum well and quantum wire structures grown on the GaAs (111)B substrates. PL intensity reduction caused by surface states was recovered remarkably by the Si ICL-based passivation.
\end{abstract}

\section{INTRODUCTION}

Quantum nanodevices are promising candidates as building blocks of next-generation LSIs because of their small sizes and ultra-low power consumption, both required for high-density integration. In particular, III-V compound semiconductor quantum devices are promising due to advanced nanotechnology for realization of sophisticated quantum confined structures, availability of variety heterostructures with defect-free heterointerfaces and superb quantum transport.

So far, GaAs-based devices have been fabricated on (001)-oriented substrates, being irrespective of whether they are semi-classical devices or quantum devices. Recent progress of III-V quantum nanotechnology, however, has revealed importance of other surfaces such as (111), (311), (411) and (521) surfaces. Furthermore, nanowire networks grown on (111)B substrates are expected to be suitable for realization of quantum logic LSIs based on the binary decision diagram (BDD) architecture[1], because the basic BDD node device requires networks having 3-fold symmetry.

The well-known difficulty is, however, that III-V surfaces possess high density of surface states which pin the surface Fermi level. This problem becomes more serious in nanostructures due to increase in the surface-to-volume ratio. Thus, success of III-V nanotechnology obviously depends on availability of a suitable surface passivation technology. To cope with this difficulty, we proposed a surface passivation scheme using an MBE-grown $\mathrm{Si}$ interlayer referred to as the $\mathrm{Si}$ interface control layer (Si ICL), and have shown that it was very effective for passivation of (001) surfaces [2-4].

In this paper, we investigate for the first time applicability of the Si ICL-based passivation scheme to the (111)B surfaces of GaAs and AlGaAs. The basic structure and band bending properties were studied by in-situ X-ray photoelectron spectroscopy (XPS) technique. Effects of surface states and the Si ICL-based passivation on nanostructure surfaces were investigated by photoluminescence (PL) study of quantum well and quantum wire structures grown on the GaAs (111)B substrates.

\section{EXPERIMENTAL}

The structures of the samples used in this study are shown in Figs.1 (a)-(d). The mole fraction of the $\mathrm{AlGaAs}$ layer was kept to be $\mathrm{Al}_{0.3} \mathrm{Ga}_{0.7} \mathrm{As}$ throughout this work. Most of the sample fabrication and 
XPS characterization steps were carried out in an ultra-high-vacuum (UHV) multi-chamber system with a common UHV transfer chamber. Samples shown in Figs. 1 (a) and (b) were planar samples prepared for basic investigation of the Si ICL-based passivation by in-situ XPS measurements. First, GaAs and AlGaAs surfaces were prepared by a standard MBE growth on (111)B substrates. Their surfaces showed well-defined Ga-stabilized $(\sqrt{19} \times \sqrt{19})$ RHEED patterns during the growth at 670 $700^{\circ} \mathrm{C}$. The patterns changed to $(2 \times 2)$ when the growth temperature was set to be lower than $500^{\circ} \mathrm{C}$. These results are in agreement with the previous works [7, 8]. In order to see the effect of surface stoichiometry, which was found crucial in the passivation of the $(001)$ surface $[3,4]$, some of the samples were irradiated with Ga flux for a few seconds at $500{ }^{\circ} \mathrm{C}$ to make the surface Ga-rich. Such Ga-irradiated surfaces showed (1x1) pattern.

Then, the MBE-grown clean planar surfaces were subjected to the Si ICL-based passivation process. The Si ICL was grown at the substrate temperature of $300^{\circ} \mathrm{C}$ for $120 \mathrm{~min}$, supplying Si atoms from the Si K-cell. During MBE growth of the Si ICL, (1x1) RHEED patterns were observed. The thickness of the Si-ICL was kept around $1 \mathrm{~nm}$. Finally the sample surface was irradiated with a nitrogen radical beam in order to partially convert the surface of Si-ICL to Si nitride by direct nitridation. This was done for 8-10 minutes at room temperature with the nitrogen flow rate of 0.4 sccm in the same MBE chamber.

At each step of the passivation process, the sample surfaces were characterized in-situ in an XPS chamber. A monochromatic $\mathrm{Al} \mathrm{K} \alpha(1486.6 \mathrm{eV})$ radiation was used as the $\mathrm{X}$-ray source. The binding energy was calibrated by setting the position of the Au4f ${ }_{7 / 2}$ peak at $84.0 \mathrm{eV}$.

After the basic XPS study, the effects of surface states and the Si ICL-based passivation on surfaces of a planar quantum well (QW) structure shown in Fig.1(c) and an embedded quantum wire (QWR) structure shown in Fig.1(d), were investigated, using photoluminescence (PL) as a probe. In both structures, the distance, $d$, between the surface and the quantum well or wire, i.e., the thickness of the upper AlGaAs barrier layer, was systematically changed by controlled anodic etching. QWR samples were prepared by our selective MBE $[5,6]$. The fabrication sequence was as follows. First, a patterned substrate having an array of mesa stripes along $\langle\overline{1} \overline{1} 2>$ direction was prepared by the electron beam lithography and wet chemical etching. Then, GaAs buffer ridge structures were grown on the substrate, followed by subsequent growth of $\mathrm{AlGaAs} / \mathrm{GaAs} / \mathrm{AlGaAs}$ structures that led to formation of embedded QWRs due to the built-in selective growth mechanism.

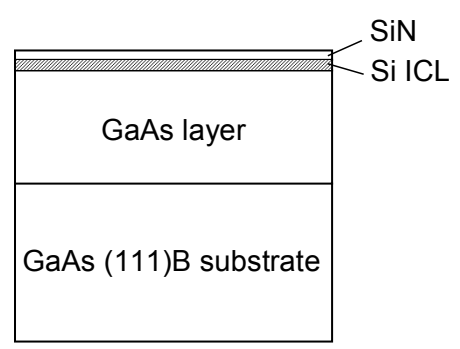

(a)

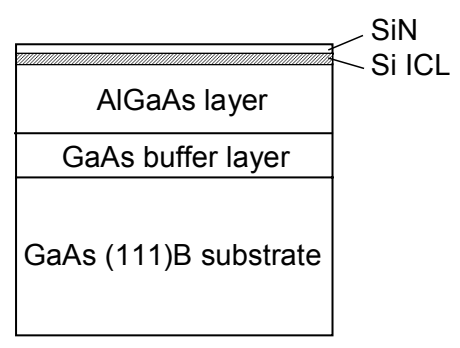

(b)

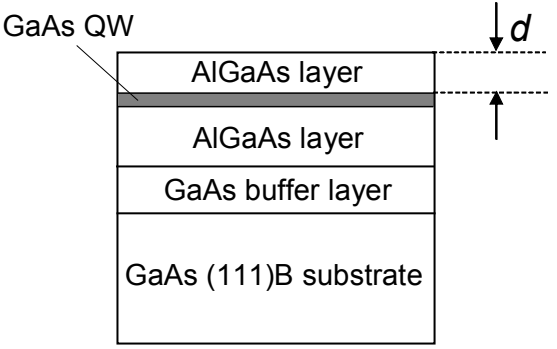

(c)

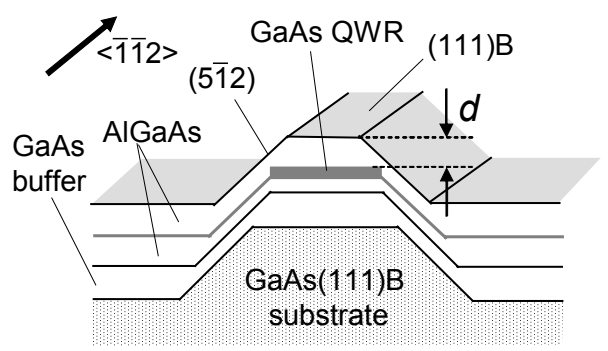

(d)

Figure 1. (a), (b); Planar sample structures for studying GaAs and AlGaAs surfaces by in-istu XPS technique. (c), (d); QW and QWR structures for PL characterization. 
The fabricated samples were characterized by a PL measurement using an $\operatorname{Ar}^{+}$laser $(\lambda=514.5$ $\mathrm{nm})$. PL peaks for fabricated QWRs and planer QWs appeared at $1.64 \mathrm{eV}$ and $1.62 \mathrm{eV}$ respectively.

\section{RESULTS AND DISCUSSION}

Successful formation of the intended passivation structure was confirmed by the in-situ XPS measurements. As an example, observed Ga3p and Si2p core level spectra are shown in Fig. 2 (a) for the MBE-grown (111)B GaAs (2x2) surface. The exit angle dependence of the spectra indicated that a uniform Si layer was formed on the GaAs surface. From the analysis of the exit angle dependence of the Ga3p peak height, the thickness of the Si ICL was determined to be $1 \mathrm{~nm}$ as intended. Similar results were obtained on other sample surfaces. Figure 2 (b) shows the measured Si2p peak for a $\mathrm{Si}$ ICL/AlGaAs (111)B sample after surface nitridation. Here, the peak was obtained by subtracting the Ga3p component. The peak could be decomposed into two components, i.e., the Si-Si bond component and the Si-N bond component. They showed a chemical shift smaller than that for $\mathrm{Si}_{3} \mathrm{~N}_{4}[9]$, which indicated formation of a Si-rich SiN layer.

At the GaAs (111)B (2x2) surface, positions of Ga3d and As 3d core level peaks indicated that the surface Fermi level, $E_{\mathrm{FS}}$, is strongly pinned at $600-800 \mathrm{meV}$ from $E_{\mathrm{V}}$. This is almost the same position with that of the chemically etched (001) surface. Thus, in spite of clear reconstruction patterns, the MBE-grown clean GaAs(111)B surface exhibits strong Fermi level pinning. This pinning persisted after irradiation of the surface with Ga flux. However, after Si ICL growth, large shifts of Ga and As core level peaks took place by nearly the same amount, indicating movement of $E_{\mathrm{FS}}$ toward $E_{\mathrm{C}}$. The magnitudes of $E_{\mathrm{FS}}$ shifts caused by Si ICL growth are summarized in Fig.3 in terms of Ga3d peak shifts for various surfaces, including that for the GaAs (001) surface taken from our previous work [3]. It is noted that the magnitude of $E_{\mathrm{FS}}$ shift for every (111)B sample was much larger than that for the (001) sample. Following our arguments on $(001)$ surface $[3,4]$, the observed $E_{\mathrm{FS}}$ shifts were concluded to be due to reduction of surface state density.

The results of PL measurements on nanostructure surfaces are summarized in Fig.4 as a function of $d$ for the QW and QWR samples with and without the Si ICL. When $d$, defined in Figs.1(c) and (d), became smaller than $50 \mathrm{~nm}$ or so, the PL intensity from the quantum confined states in the embedded QWs and QWRs rapidly reduced exponentially for the unpassivated samples. This is due to the nonradiative surface recombination triggered by the carrier transfer from quantum confined states to surface states via tunneling $[6,10]$. By applying the Si ICL method, a large increase in PL intensity was seen for a QWR sample as seen in Fig.4. This can be explained by large reduction of surface states.

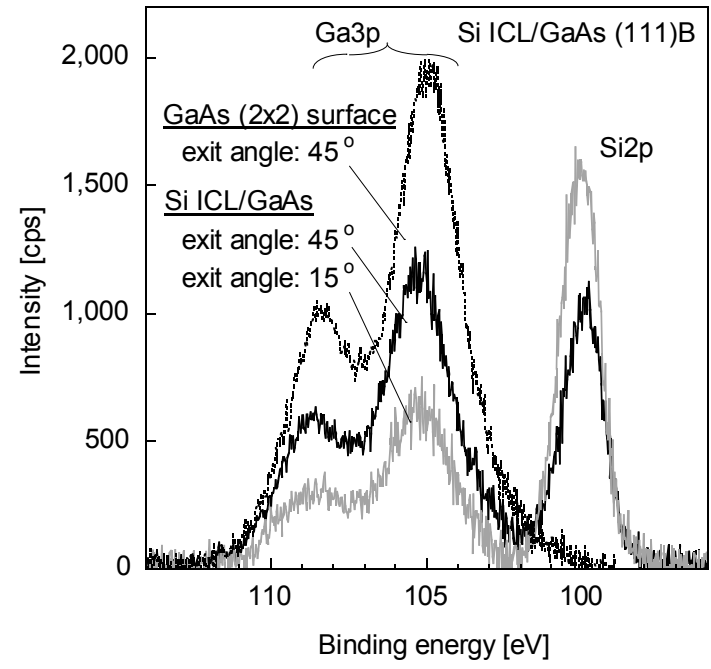

(a)

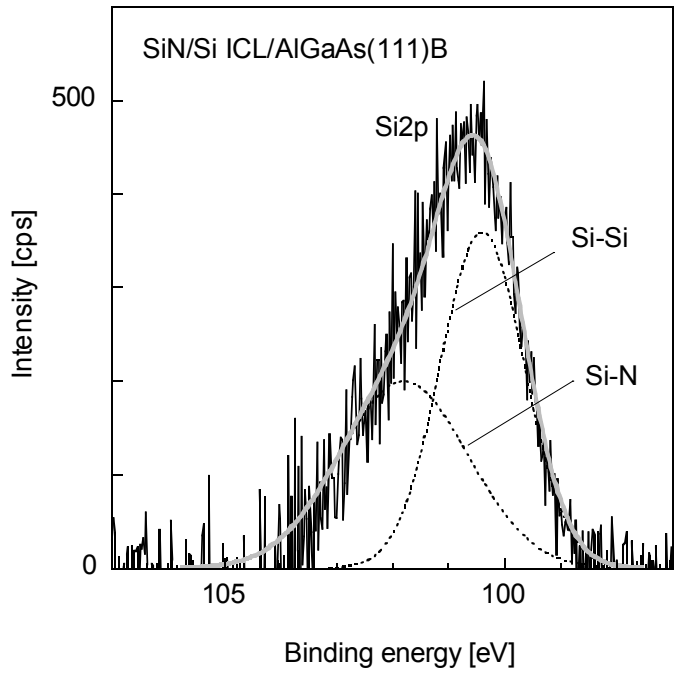

(b)

Figure 2. (a) XPS Ga3p and Si2p spectra before and after Si ICL formation onto GaAs (111)B (2x2) surface and (b) Si2p spectra from the Si ICL/AlGaAs (111)B sample after the surface nitridation. 


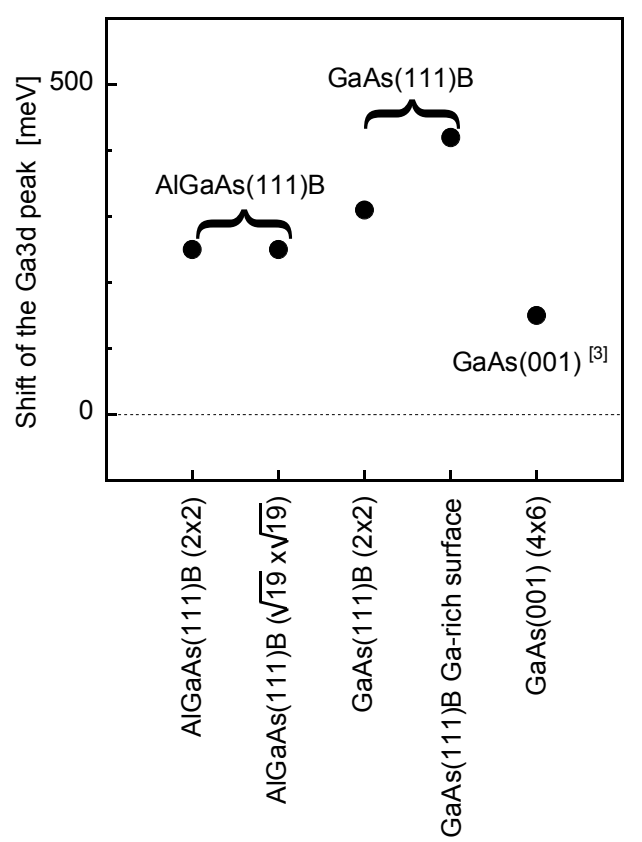

Figure 3. The magnitude of the Ga3d peak shift by Si ICL growth for several surfaces.

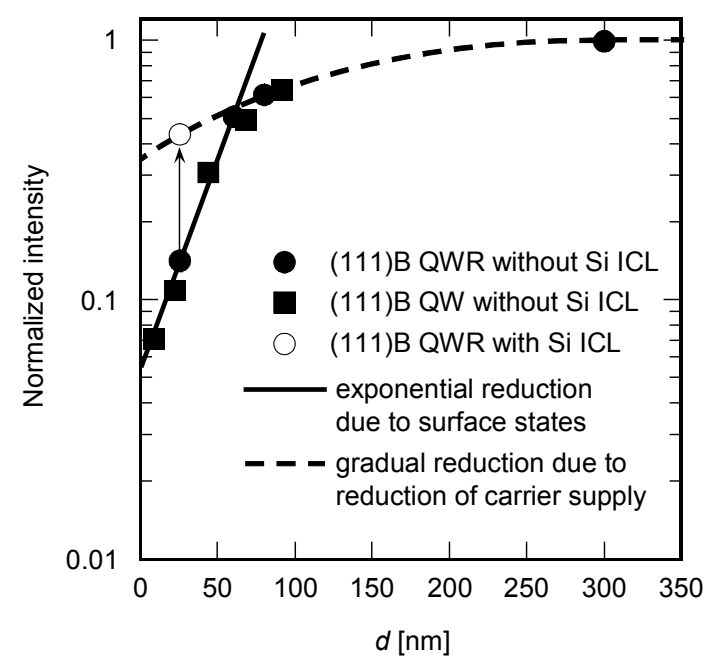

Figure 4. Plot of the normalized PL intensity for QWs and QWRs with and without the SiICL-based passivation. $d$ is the distance between the surface and QW or QWR shown in Fig.1(c) and (d).

\section{CONCLUSION}

In this paper, we investigated applicability of the Si ICL-based surface passivation scheme to GaAs and AlGaAs (111)B surfaces. An in-situ XPS study confirmed formation of the intended passivation structure. Large shifts of the surface Fermi level position were observed for the (111)B surfaces after Si ICL growth, with magnitudes larger than that for the (001) surface. PL measurements were also used to examine the effectiveness of the Si ICL-based passivation on the surfaces of the AlGaAs/GaAs quantum well and quantum wire structures grown on the GaAs (111)B substrates. PL intensity reduction caused by surface states was recovered remarkably by the Si ICL-based passivation. These results indicated that the Si ICL-based passivation scheme was effective for surface passivation of the GaAs and AlGaAs (111)B surfaces of the quantum nanostructures.

\section{Acknowledgments}

This work is supported partly by 21 Century COE program, "Meme-media Technology Approach to the R\&D of next-generation ITs," at Hokkaido University and partly by Grant-in-Aid for Scientific Research (C)-16560288 both from the Japanese Government.

\section{References}

[1] H. Hasegawa, Appl. Surf. Sci. 212 - 213 (2003) pp. 311-318.

[2] H. Hasegawa, Thin Solid Films 367 (2000) pp. 58-67.

[3] Y. Nakano, N. Negoro and H. Hasegawa, Jpn. J. Appl. Phys. 41 (2002) pp. 2542-2547.

[4] S. Anantathanasarn and H. Hasegawa, Appl. Surf. Sci. 216 (2003) pp. 275-282.

[5] I. Tamai, T. Sato and H. Hasegawa, Jpn. J. Appl. Phys. 44 (2005) pp. 2652-2656.

[6] N. Shiozaki, T. Sato and H. Hasegawa, J. Vac. Sci. Technol. B 23 (2005) pp.1714-1721.

[7] D. K. Biegelsen, R. D. Bringans, J. E. Northrup and L.-E. Swartz, Phys. Rev. Lett. 65 (1990) pp. 452-455.

[8] D. A. Woolf, D. I. Westwood and R.H. Williams, Appl. Phys. Lett. 62 (1993) pp. 1370-1372.

[9] C. H. F. Peden, J. W. Rogers, N. D. Shinn, K. B. Kidd and K. L. Tsang, Phys. Rev. 47 (1993) pp. $15622-15629$.

[10] .Z. Sobiesierski, D. I. Westwood, D. A. Woolf, T. Fukui and H. Hasegawa, J. Vac. Sci. Technol. B 11 (1993) pp. 1723-1726. 